professionals registering on an online platform to receive a panel with four dried tube specimens with unknown reactivity for syphilis. The respondents test the samples as if it they were from their own patients and submit the results online for subsequent analysis.

Methods We analysed reports from eight EQA-RT rounds conducted in 2014-2016. The quality of the professionals' performance was assessed by examining the degree of concordance between the results from the panel samples that were expected and those actually reported. A certificate of approval is issued in the event of $70 \%$ accuracy. Any professional receiving less than $70 \%$ approval rating is sent a report suggesting possible causes and solutions for issues that might have been responsible to prevent the quality of testing.

Results The average number of participants per round was 843 (545 in 2014, 909 in 2015 and 1.075 in 2016) distributed in 347 mainly primary healthcare units. It was interesting to note that many higher complexity services, despite registering in the program, failed to report the results (e.g. a 55\% abstention rate in one of the rounds). Regarding the performance on EQA-RT, an average of $93 \%$ of respondents were approved per round, with $88 \%$ achieving 100\% accuracy. The most common failures identified so far have been incorrect pipetted sample volumes and result reading times

Conclusion The majority of healthcare professionals currently participating in Brazil's EQA-RT program are maintaining a high level of RT quality. While the number of participants doubled in 2014-2016, there is still a need for education work to encourage more adherence to the Program aimed at ensuring the reliability and credibility of the TR results.

\section{P6.18 DISTANCE LEARNING COURSE ABOUT RAPID TEST FOR HEALTHCARE PROFESSIONALS AS AN IMPORTANT STRATEGY TO INCREASE THE ACCESS TO SYPHILIS DIAGNOSIS}

'Pâmela Cristina Gaspar, 'Regina Aparecida Comparini, 'Ana Flávia Pires, ${ }^{1}$ Miriam Franchini, ${ }^{1}$ Adele Schwartz Benzaken, ${ }^{2}$ Lucy Maria Bez Birolo Parucker. ${ }^{1}$ Department of Surveillance, Prevention and Control of STI, HIVIAIDS and Viral Hepatitis, Brasilia, Brazil; ${ }^{2}$ Federal University of Santa Catarina, Florianópolis, Santa Catarina, Brazil

\subsection{6/sextrans-2017-053264.669}

Introduction Since 2015, Brazil has had a worrying epidemic of syphilis. From 2014 to 2015 there was a growth of $21 \%$ of congenital syphilis, reaching 19228 cases in 2015. The reduction of mother-to-child transmission in 2017 is a priority goal of the government. Increasing access to diagnosis through rapid testing (RT) is one of the key strategies established to achieve this goal. Only in 2016, more than 6 million RT were distributed by the Ministry of Health $(\mathrm{MoH})$ to healthcare facilities in the country. In addition, it is also necessary to increase the number of healthcare professionals capable to perform the RT. Considering the continental dimension of the country and the need for an alternative to on-site trainings, $\mathrm{MoH}$ offers a free distance learning course called TELELAB. We present the extent of the coverage of this education strategy.

Methods TELELAB provides online courses about diagnostic of sexually transmitted infection with video lessons and instruction manuals. After the course is completed, professionals are awarded a certificate once they pass an exam with $70 \%$ or higher. We analysed TELELAB's database regarding the number of healthcare professionals who obtained certification in "Syphilis diagnose course" during 2016, their professions and city of residence.

Results In 2016, 7064 healthcare professionals were certified. Nursing is the profession with the highest number of certification (i.e. $72 \%$ of the total). 842 different municipalities were observed. Out of this, 4811 healthcare professionals (i.e. $68 \%$ of the total) reside out of capital, and many of them in remote and rural areas with no access to laboratory services.

Conclusion TELELAB is an important strategy to ensure greater access to syphilis diagnosis, since it better qualifies healthcare professionals, especially nurses, that are present in major basic care services and usually diagnoses and treat the patient. Considering that Brazil has 5561 municipalities, there is still need to widespread this modality of distance learning course and incentive for higher adherence.

\section{P6.19 KNOWLEDGE, ATTITUDE AND PRACTICE ABOUT SEXUALLY TRANSMITTED DISEASES AMONG UNIVERSITY STUDENTS IN GHANA}

R Acheampong. Kingdom Impart Church INT.L, Accra, Ghana

\subsection{6/sextrans-2017-053264.670}

Introduction Sexually transmitted diseases (STDs) remain an important cause of morbidity and mortality among women in the child-bearing age. In order to institute appropriate preventive measures there is need to establish the profile of knowledge of the predisposing factors and causation of STDs, attitude to sexual practice and sexual patterns among the susceptible young people, such as university students.

Method A detailed questionnaire identifying socio-demographic characteristics, sexual patterns, knowledge of STDs as well as attitudes towards prevention of STDs was administered to 400 non-medical students of University Of Cape Coast,Ghana.

Results Knowledge of the clinical features of gonorrhoea and AIDS was high; most knew the predisposing factors for STDs (multiple sexual partners 90\%; unprotected sexual intercourse 93\%; rape $81 \%$; sex outside marriage $78 \%$, and sex under the influence of alcohol $73 \%$ ) but not so for syphilis. Males were three times more likely to contract STDs (27\%) than their female $(9 \%)$ counterparts. Whereas knowledge on methods of prevention was high $(>90 \%)$ it was not followed by appropriate behavioural patterns. More female (33.5\%) students had heard about Trichomonas vaginalis than males (23\%); $(X 2=17.1 ;<0.0001)$. This study has shown that more female than male students got information from their parents $(\mathrm{X} 2=25.3 ; \mathrm{p}<0.001)$ while more male students had their information from previous sexual intercourse $(\mathrm{X} 2=12.9$; $\mathrm{p}=0.001)$.

Conclusion The level of knowledge about STDs and their prevention is not matched by sexual behavioural patterns, and male students undertake more risky sexual behaviour. Sexual education should be introduced at the university as a means of increasing students' awareness about the problem and prevention of sexually transmitted diseases including HIV/AIDS. 